\title{
Caso de desafío diagnóstico: Fístula dural
}

\section{Schneider M. ${ }^{1}$, M. Hirsch S. ${ }^{2}$, P. Paredes M. ${ }^{3}$}

1. Residente de Radiología, Clínica Alemana, Universidad del Desarrollo. Santiago -Chile.

2. Radiólogo, Departamento de Imágenes, Clínica Alemana de Temuco. Temuco-Chile.

3. Neurorradiólogo, Departamento de Imágenes, Clínica Alemana de Santiago. Santiago-Chile.

\section{Diagnostic challenge case: Dural fistula}

\section{Resumen:}

Se presenta el caso de una paciente de sexo femenino, de 69 años, que refiere tinnitus pulsátil de dos meses de evolución percibido en la región retroauricular derecha. Se presentan los hallazgos en estudios de imágenes, diagnóstico y se presenta una corta revisión del tema.

Palabras clave: Fístula dural arteriovenosa, Malformaciones del sistema venoso central, Tinnitus pulsátil.

\begin{abstract}
:
We present the case of a female patient, 69 years old, who reported pulsatile tinnitus since two months ago perceived in the right retroauricular region. We present the findings in image studies, diagnosis and a short review about the topic.

Keywords: Dural Arteriovenous Fistula, Central Nervous System Vascular Malformations, Pulsatile Tinnitus.
\end{abstract}

\section{Historia Clínica}

Paciente de 69 años, sexo femenino. Consulta a otorrinolaringólogo por historia de 2 meses de evolución consistente en tinnitus pulsátil percibido en la región retroauricular derecha, como parte de su estudio se le solicita ecotomografía Doppler color de vasos cervicales para lo cual acude a nuestro Departamento de Imágenes (Figura 1).

¿Cuál es su diagnóstico?
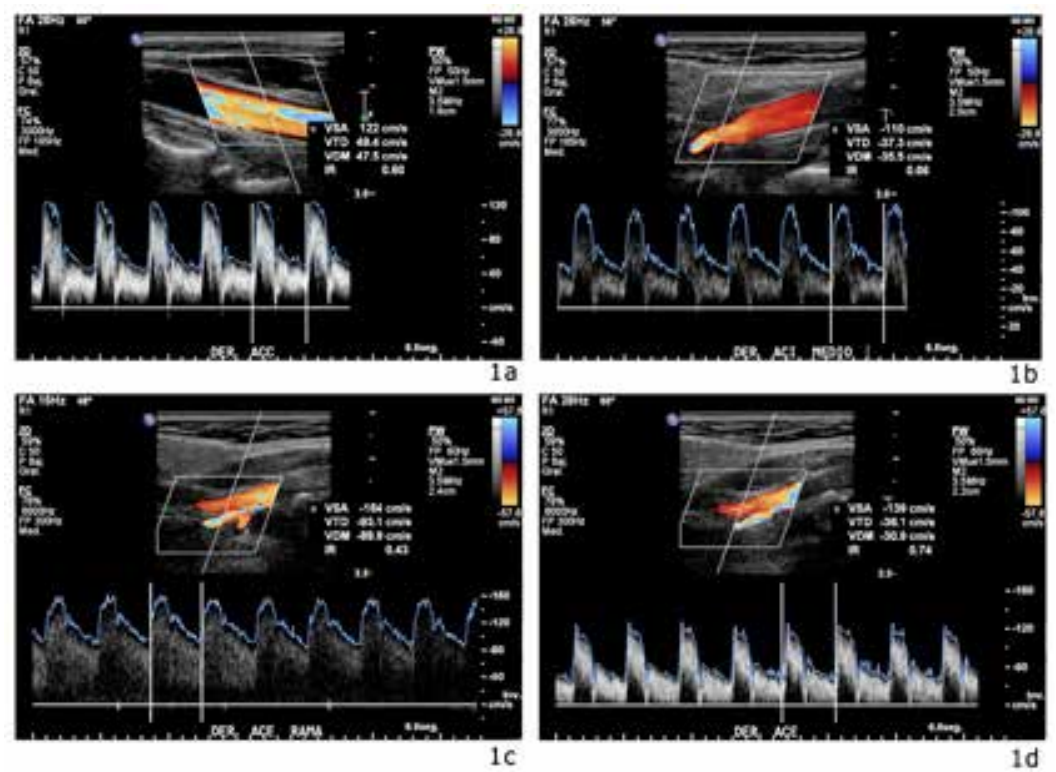


\section{Resolución del caso}

Se complementa estudio con AngioRM (Figura 2) que demuestra un conglomerado de vasos anómalos que ocupan el canal del hipogloso derecho y se asocian a señal de flujo arterializado en la vena yugular interna correspondiente, compatible con fístula arteriovenosa dural. Los hallazgos fueron confirmados mediante angiografía con sustracción digital.
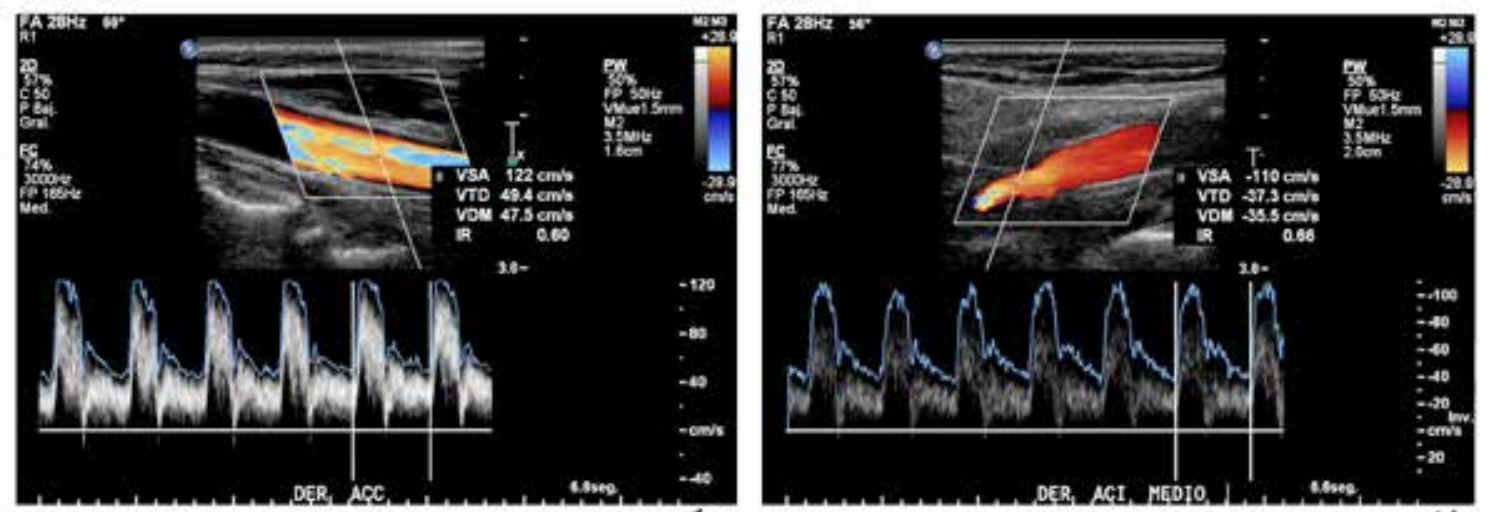

$1 \mathrm{a}$

$1 \mathrm{~b}$
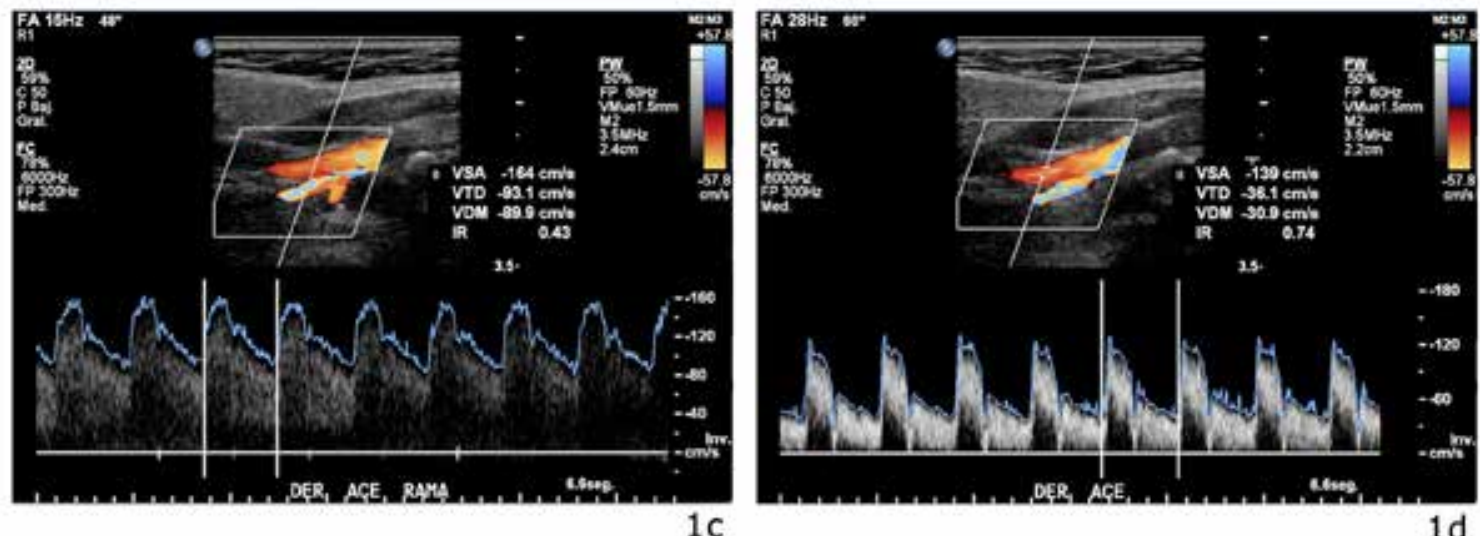

Figura 1: Ultrasonido Doppler de arteria carótida común derecha (a), carótida interna derecha (b), rama posterior de carótida externa $(A C E)$ (c) y ACE (d). Destaca flujo de baja resistencia en rama de ACE y fenómeno de aliasing en dicha rama por mayor velocidad del flujo.
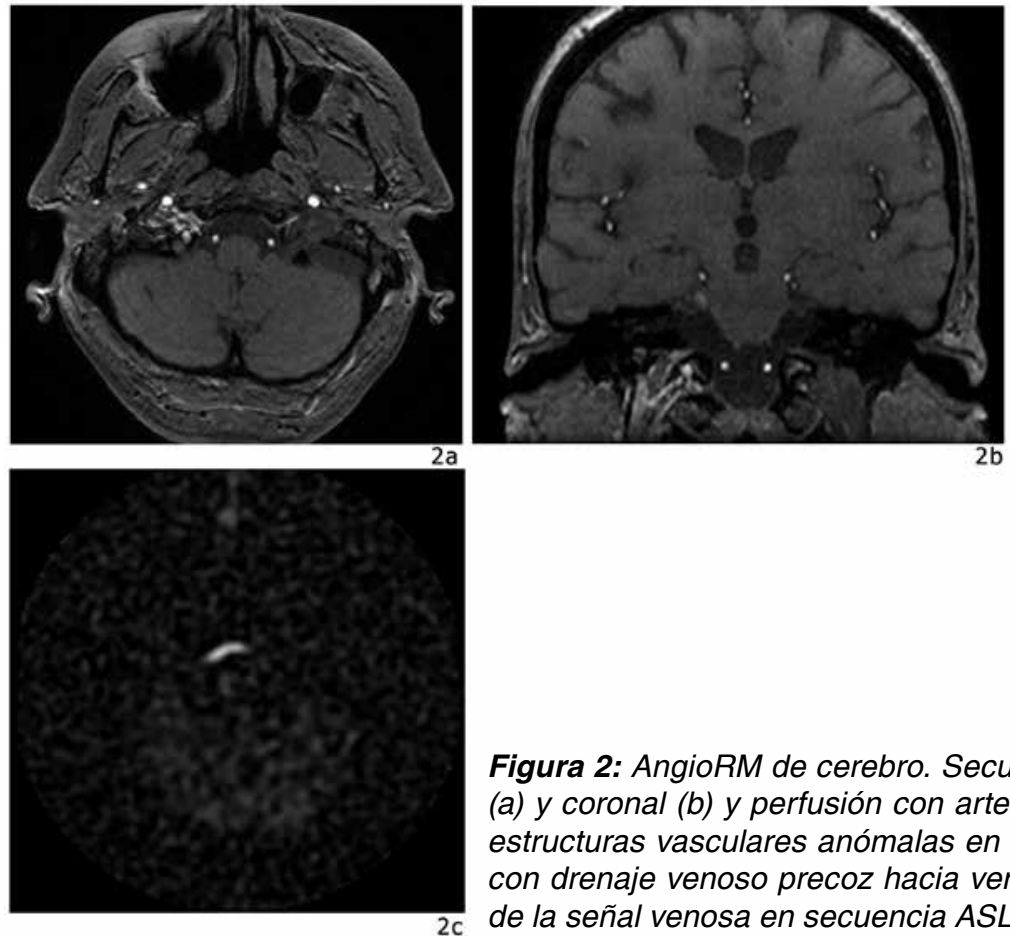

Figura 2: AngioRM de cerebro. Secuencias time of flight (TOF) arterial axial (a) y coronal (b) y perfusión con arterial spin labeling (ASL) (c). Destacando estructuras vasculares anómalas en relación a conducto hipogloso derecho con drenaje venoso precoz hacia vena yugular interna ipsilateral y aumento de la señal venosa en secuencia ASL. 


\section{Diagnóstico}

Fístula arteriovenosa dural.

\section{Discusión}

Las fístulas arteriovenosas durales (FAVD) son comunicaciones anormales entre las arterias meníngeas y los senos venosos, venas meníngeas o venas corticales. Su localización más frecuente es la pared o alrededor de los senos venosos ${ }^{(1,2,3,4)}$ Dural arteriovenous fistula (DAVF y dan cuenta un 10-15\% de todas las malformaciones vasculares intracraneales. Han sido reportadas en todos los grupos etarios, siendo más frecuentes en la quinta y sexta década de la vida, su incidencia estimada es de 0.17 casos por 100.000 habitantes, algo mayor en mujeres en razón de 2:1(2,5,6).

La gran mayoría de las FAVD del adulto son adquiridas, aunque también se han reportado fístulas durales congénitas ${ }^{(3)}$.

Las FAVD se diferencian de las malformaciones arteriovenosas piales (MAV) en que estas últimas son lesiones tienen un nido vascular intraaxial y que reciben aporte arterial de arterias piales, sin identificarse un lecho capilar.

Su etiología y fisiopatología no se conocen completamente. La gran mayoría se consideran idiopática, existiendo un grupo que tiene documentado como gatillante un trauma, craneotomía previa, tumores o trombosis de un seno venoso dural. Las FAVD van produciendo estenosis progresiva u oclusión de un seno venoso dural. A medida que aumenta la presión venosa se desarrolla hipoperfusión focal crónica, lo que desencadena la producción de factor-1a que aumenta la expresión del factor de crecimiento vascular endotelial, el que a su vez desencadenaría la angiogénesis con que las arterias meníngeas desarrollarían conexiones fistulosas con el seno dural o venas corticales. Otra teoría consiste en que las comunicaciones arteriovenosas fisiológicas entre las arterias meníngeas y senos venosos durales, crecen en respuesta a un aumento de la presión venosa local, resultando en el desarrollo de un shunt patológico(2,4,7).

La historia natural de la enfermedad es altamente variable, pudiendo evolucionar desde la resolución espontánea hasta una hemorragia fatal. Las manifestaciones clínicas dependen en gran medida del patrón de drenaje venoso y de la localización de la $F A V D^{(1,2,4)}$. El tinnitus pulsátil es un síntoma común particularmente en lesiones relacionadas al seno transverso o sigmoideo. Las FAVD del seno cavernoso (fístula carótido-cavernosa) pueden presentar oftalmoplejia, proptosis, quemosis, dolor retro-orbitario y disminución de la agudeza visual. Otras presentaciones severas de las fístulas durales incluyen hemorragia intracraneal y déficits neurológicos no hemorrágicos como convulsiones, parkinsonismo, síntomas cerebelosos $y$ anormalidades en los pares craneanos $^{(3,4)}$.
Se han propuesto múltiples clasificaciones, siendo las más utilizadas actualmente las de Borden y Cognard (Tabla 1). La clasificación de Borden se basa en el sitio de drenaje venoso y la ausencia o presencia de drenaje venoso cortical. La clasificación de Cognard incorpora información sobre la dirección del flujo en el seno dural, además de la presencia o ausencia de ectasia venosa en las venas corticales comprometidas ${ }^{(1)}$. Ambas se basan en el principio de que las fístulas con reflujo venoso cortical o drenaje directo a una vena cortical dse asocian a peor pronóstico(2). A mayor grado en ambas clasificaciones, hay mayor riesgo que la fístula sea sintomática y que se asocie con hemorragia ${ }^{(1)}$.

Tabla 1. Clasificaciones de las fistulas durales arteriovenosas intracraneales ${ }^{(1)}$.

\begin{tabular}{|c|c|}
\hline \multicolumn{2}{|c|}{ Clasificación de Borden } \\
\hline I & : Drenaje venoso en un seno dural. \\
\hline II & $\begin{array}{l}\text { Drenaje venoso en un seno dural con } \\
\text { drenaje venoso cortical (DVC). }\end{array}$ \\
\hline III & $\begin{array}{l}\text { Drenaje venoso directamente en una vena } \\
\text { subaracnoidea (sólo DVC) }\end{array}$ \\
\hline \multicolumn{2}{|c|}{ Clasificación de Cognard } \\
\hline I & $\begin{array}{l}\text { : Drenaje venoso en un seno dural con flujo } \\
\text { anterógrado normal. }\end{array}$ \\
\hline Ila & $\begin{array}{l}\text { : Drenaje venoso en un seno dural con flujo } \\
\text { retrógrado. }\end{array}$ \\
\hline Illb & $\begin{array}{l}\text { : Drenaje venoso en un seno dural con flujo } \\
\text { anterógrado normal y DVC. }\end{array}$ \\
\hline \multicolumn{2}{|c|}{$\begin{array}{l}\text { Ila }+ \text { b: Drenaje venoso en un seno dural con flujo } \\
\text { retrógrado y DVC. }\end{array}$} \\
\hline III & $\begin{array}{l}\text { : Drenaje venoso directo en una vena suba } \\
\text { racnoidea (sólo DVC). }\end{array}$ \\
\hline IV & $\begin{array}{l}\text { Drenaje venoso directo en una vena suba } \\
\text { racnoidea con ectasia venosa. }\end{array}$ \\
\hline V & $\begin{array}{l}\text { Drenaje venoso directo en las venas es } \\
\text { pinales perimedulares. }\end{array}$ \\
\hline
\end{tabular}

Debido a que el shunt desata cambios hemodinámicos en las arterias que alimentan la fístula (con mayor frecuencia las arterias carótidas externas, internas y vertebrales), el ultrasonido Doppler puede ser una buena herramienta para el screening y seguimiento. Las características al Doppler de las arterias fuente tales como un bajo índice de resistencia, aumento del volumen de flujo, aumento de la velocidad de peak sistólico y aumento de la velocidad de fin de diástole son útiles para sospechar una $F A V D^{(8)}$, por lo que es muy importante buscar dirigidamente estos hallazgos.

La tomografía computada (TC) sin contraste sólo puede demostrar las secuelas de las manifestacio- 
nes agresivas de una FAVD, como son hemorragia o edema cerebral ${ }^{(2,4,6)}$. El patrón de distribución de la hemorragia detectada en una TC o en resonancia magnética (RM) no es especifico, siendo el más comúnmente detectado el patrón intraparenquimatoso lobar. Menos frecuentemente se puede ver hemorragia subaracnoidea, especialmente en la categoría $V$ de Cognard. Algunas veces se distinguen hematomas subdurales, los que han sido reportados en FAVD de fosa craneal anterior ${ }^{(1)}$. La angiografía por TC puede detectar arterias aferentes de mayor calibre, venas de drenaje dilatadas, vasos prominentes en los senos durales o meninges, trombosis venosa cerebral y aneurismas $^{(2,8)}$.

Las secuencias angiográficas de RM también permiten visualizar estas características anatómicas de las FAVD con alta sensibilidad y especificidad ${ }^{(2)}$. La RM tiene dos grandes formas para medir la perfusión cerebral, la primera es por medio de secuencias con administración de contraste endovenoso basado en gadolinio, tales como DSC (dynamic susceptibility contrast-enhanced) y DCE (dynamic contrast-enhanced), la segunda se denomina ASL (arterial spin labeling) y no requiere uso de contraste endovenoso, ya que utiliza el agua de la sangre arterial marcada magnéticamente como medio de contraste endógeno(10). EI estudio de perfusión ASL puede ser un marcador muy sensible en la evaluación del flujo vascular alterado en el contexto de una FAVD, identificando alta señal en las ubicaciones de estructuras venosas mayores o venas corticales, siendo especialmente útil en el screening de éstas lesiones o pequeñas malformaciones arteriovenosas ${ }^{(11)}$, aunque para algunos autores esto sigue siendo motivo de controversia(6).

Las secuencias de susceptibilidad magnética permiten ver el shunt arteriovenoso como hiperintensidad de la señal venosa, relacionada al wash-in rápido de la sangre oxigenada; también pueden representar adecuadamente el drenaje venoso cortical retrógrado asociado a la FAVD demostrando venas superficiales dilatadas en el hemisferio cerebral ipsilateral, así como también venas de drenaje profundo prominentes ${ }^{(6)}$.

En la secuencia 3D TOF (Three dimensional Time of Flight) la identificación de hiperintensidades nodulares o curvilíneas alrededor de un seno dural o cerca de venas cerebrales se describe como un hallazgo clave para sospechar de una FAVD ${ }^{(12)}$. Por último, la técnicas angiográficas $4 \mathrm{D}$ con contraste han demostrado ser una alternativa confiable para la caracterización de las FAVD intracraneales, especialmente con respecto al sitio de fístula y vía de drenaje venoso ${ }^{(13)}$.

La angiografía con sustracción digital es el estándar de referencia para el diagnóstico, permitiendo detectar el llene precoz de las estructuras asociadas al shunt. Su alta resolución temporal y habilidad de representar ramas arteriales especificas le permiten identificar y clasificar las FAVD con excelente precisión ${ }^{(2)}$. También permite reconocer importantes características de la fístula como la presencia de drenaje venoso cortical, obstrucción al retorno venoso y aneurismas arteriales o venosos; siendo esto esencial para planificar el manejo(1).

El tratamiento de las FAVD depende del tipo de fístula, riesgos asociados a tratamiento, además de los síntomas y preferencias del paciente. El seguimiento y observación se consideran una opción en las FAVD de bajo riesgo. La embolización endovascular transarterial o transvenosa es actualmente la primera línea de tratamiento para las FAVD agresivas, así mismo es una opción en aquellas de bajo riesgo sintomáticas o con riesgo de volverse agresivas. La cirugía se reserva para los casos en que no se puede realizar la terapia endovascular ${ }^{(2,14)}$. La radiocirugía se considera una alternativa poco invasiva para aquellas FAVD benignas, con nidus pequeño o para aquellos que no son candidatos a otros tratamientos. Su desventaja es la latencia entre el tratamiento y su efecto de al menos 6 meses $^{(3,14)}$.

\section{Referencias}

1. Serulle Y, Miller TR, Gandhi D. Dural Arteriovenous Fistulae: Imaging and Management. Neuroimaging Clin N Am 2016; 26: 247-258.

2. Tsai L-K, Liu H-M, Jeng J-S. Diagnosis and management of intracranial dural arteriovenous fistulas. Expert Rev Neurother 2016; 16: 307-318.

3. Paredes I, Martinez-Perez R, Munarriz PM, CastañoLeon AM, Campollo J, Alén JF, et al. Fístulas durales arteriovenosas intracraneales. Experiencia con 81 casos y revisión de la literatura. Neurocirugia 2013; 24: 141-151.

4. Gandhi D, Chen J, Pearl M, Huang J, Gemmete JJ, Kathuria S. Intracranial dural arteriovenous fistulas: classification, imaging findings, and treatment. American J Neuroradiol 2012; 33: 1007-1013.

5. Jabbour P, Tjoumakaris S, Chalouhi N, Randazzo C, Gonzalez LF, Dumont A, et al. Endovascular treatment of cerebral dural and pial arteriovenous fistulas. Neuroimaging Clin N Am 2013; 23: 625-636.

6. Mossa-Basha M, Chen J, Gandhi D. Imaging of Cerebral Arteriovenous Malformations and Dural Arteriovenous Fistulas. Neurosurg Clin N Am 2012; 23: 27-42.

7. Reynolds MR, Lanzino G, Zipfel GJ. Intracranial Dural Arteriovenous Fistulae. Stroke 2017; 48: 1424-1431.

8. Tsai L, Jeng J, Yip P. Ultrasonography in Intracranial Dural Arteriovenous Fistula. J Med Ultrasound 2008; 16: 57-64.

9. Lee C-W, Huang A, Wang Y-H, Yang C-Y, Chen Y-F, Liu $\mathrm{H}-\mathrm{M}$. Intracranial Dural Arteriovenous Fistulas: Diagnosis and Evaluation with 64-Detector Row CT Angiography. Radiology 2010; 256: 219-228.

10. Essig M, Shiroishi MS, Nguyen TB, Saake M, Provenzale JM, Enterline D, et al. Perfusion MRI: the five most frequently asked technical questions. Am J Roentgenol 2013; 200: 24-34.

11. Fischbein NJ, Rosenberg J. Identification of Venous Signal on Arterial Spin Labeling Improves Diagnosis of Dural Arteriovenous Fistulas and Small Arteriovenous 
Malformations. Am J Neuroradiol 2012; 33: 61-68.

12. Meckel S, Maier M, Ruiz DSM, Yilmaz H, Scheffler K, Radue EW, et al. MR angiography of dural arteriovenous fistulas: diagnosis and follow-up after treatment using a time-resolved 3D contrast-enhanced technique. Am J Neuroradiol 2007; 28: 877-884.

13. Nishimura S, Hirai T, Sasao A, Kitajima M, Morioka M,
Kai Y, et al. Evaluation of dural arteriovenous fistulas with 4D contrast-enhanced MR angiography at 3T. Am J Neuroradiol 2010; 31: 80-85.

14. Paul AR, Colby GP, Huang J, Tamargo RJ, Coon AL. Selection of Treatment Modalities or Observation of Dural Arteriovenous Fistulas. Neurosurg Clin N Am 2012; 23: 77-85.

Schneider D, et al. Caso de desafío diagnóstico: Fístula dural. Rev Chil Radiol 2018; 24(3): 112-116.

Correspondencia: Daniel Schneider M. / dschneider@alemana.cl

Trabajo enviado el 03 de marzo de 2018. Aceptado para publicación el 26 de septiembre de 2018. 